\title{
REHABILITACIÓN ACÚSTICA DE UN AULA DE MÚSICA
}

\author{
Paloma Pérez Castillo \\ Universidad de La Rioja
}

\begin{abstract}
RESUMEN: Las aulas de música carecen, por lo general, de unas condiciones acústicas idóneas. Mediante un análisis de variables perceptivas y de parámetros físicos, se han podido identificar aquellos valores acústicos que garantizan el confort acústico deseado. Tomados estos valores como criterio para la rehabilitación acústica de un aula de música previamente estudiada, la mejora alcanzada resulta altamente satisfactoria. Con todo ello se pretende contribuir a una mayor efectividad de la comunicación oral y musical para el buen desarrollo de la didáctica de la música.
\end{abstract}

ABSTRACT: Generallly, music in classrooms lacks apropiate acoustic characteristics. With the help of an análisis of perceptive variables and plysical parameters, those acoustic values which guarantee the acoustic confort have been able identify. These values taken like a criteria to the acoustic restoration of a music classroom sutied before, the reached improvement results satisfactory. With all ol them it pretends to contribute to a better effectiveness of the oral and musical communication for a good development of the music didactics.

PALABRAS CLAVE: Condiciones acústicas, comunicación, sensibilización, didáctica de la música.

KEYWORDS: Acoustic conditions, communication, sensitiveness, music didactics.

\section{INTRODUCCIÓN}

Buscamos lugares idóneos y confortables para las diferentes actividades y funciones que llevamos a efecto durante nuestra vida. Tratamos de acondicionar nuestro entorno y nuestros locales para que podamos realizar los trabajos y las actividades de una manera efectiva y gratificante. Si focalizamos estas necesidades a las aulas, bien patentes son las conveniencias de una buena luminosidad, adecuada ventilación, etc., y obviamente es de muy vital importancia satisfacer las adecuadas condiciones acús- 
ticas: consideremos la gran incidencia de las señales acústicas (comunicación oral, actividades musicales, etc.) que entran en juego en la actividad docente.

Muchas veces lo obvio no se toma con la consideración debida; y precisamente por ser obvio, quizás se nos antoja, frecuentemente, que ciertas problemáticas no necesitan de nuestra intervención, como si fueran problemas que se van a arreglar por sí mismos. Pero no, y aunque ciertamente hay aulas en las cuales, considerando las condiciones acústicas, la actividad docente resulta satisfactoria, hay muchas en las que la comunicación oral y las actividades musicales resultan realmente penosas.

En este artículo se describen algunos fundamentos sobre la incidencia de las condiciones acústicas en la comunicación docente así como en otras actividades que se desarrollan en el aula. Es manifiesto que el profesor hace un esfuerzo por hacerse entender, un esfuerzo por una comunidad inteligible; si las condiciones acústicas no son adecuadas, el alumno pierde irremediablemente parte del mensaje docente, y asimismo resulta una merma de efectividad si se trata de actividades de grupo (musicales por ejemplo). Si consideramos el alto número de personas, profesores y alumnos ligados a la actividad en el aula y la gran cantidad de horas dedicadas a ello, tengo que concluir que el acondicionamiento acústico de las aulas es una importante cuestión; no podemos negar que es una cuestión de muy notable incidencia social.

Entiendo que es importante hacer una Ilamada de atención hacia las deficientes normativas que actualmente existen; dicho de otra manera, satisfaciendo todos los requisitos legales actuales sobre las aulas, pueden resultar, bajo el ángulo de confort acústico, verdaderamente problemáticas. De esta manera, las propuestas que se hacen sobre normativas, vienen a paliar patentes deficiencias a estos respectos.

\section{Metodología}

A continuación se expone el procedimiento llevado a cabo en la rehabilitación acústica de un aula. El primer paso fue tratar de conocer las aulas de música de los Institutos de Educación Secundaria de La Rioja para escoger una muestra característica. Una vez seleccionadas las aulas, el segundo paso fue hacer un estudio acústico en profundidad de las cuatro aulas de música escogidas como representativas del resto de aulas. Se estudia su ubicación en el centro, sus dimensiones, características y se toman notas de las primeras impresiones acústicas de las mismas apreciando aspectos positivos y negativos.

Con ayuda de la dirección de estos centros, de sus profesores de música y del alumnado se procede a realizar dos tipos diferentes de pruebas; una de tipo vivencial con participación activa de los alumnos de cada centro, a través de mediciones de tipo psicoacústico; y otra de tipo físico con la instrumentación adecuada y con ayuda de un especialista en dicha materia, a través de mediciones de tipo físicoacústico (Delgado, 1995).

Las pruebas de tipo vivencial se llevan a cabo mediante el Test de Inteligibilidad de la palabra y mediante una encuesta. El material utilizado en el test consiste en cien palabras bisílabas equilibradas de acuerdo con la distribución existente en el idioma español, pero formando palabras sin sentido (Alonso, 2000). Las cien palabras están agrupadas en diez grupos de diez palabras. 
Las mediciones físico-acústicas se llevan a cabo por medio de un analizador de Fourier (tipo 2034 de la Brüel \& Kjaer y el 2800 de la Larson Davis con un micrófono) (Pierce, 1985). Los parámetros físicos medibles en este estudio son:

- El nivel sonoro de los bloques de palabras: es el nivel de presión sonora expresado en $\mathrm{dB}(\mathrm{A})$.

- El nivel de ruido: es el nivel de presión sonora del ruido de fondo expresado en $\mathrm{dB}(\mathrm{A})$.

- El tiempo de reverberación, definido como el tiempo que tarda en decaer una señal estacionaria sesenta decibelios una vez cesada la fuente.

- EI RASTI (Rapid Speech Index), que es un índice rápido de transmisión de la palabra que se basa en un sistema de modulación limitado a dos bandas de octava, a 500 y 2.000 hercios.

- La Claridad acústica, definida como la relación de la energía sonora que nos llega entre los primeros 50 milisegundos y la total.

- El tiempo de decaimiento primario (E.D.T.), que es el tiempo que tarda un pulso en decaer diez decibelios.

- El tiempo de elevación, definido como el tiempo que tarda en llegar el cincuenta por ciento de la energía sonora generada por un pulso (Kinsler y Frey, 1988).

\section{Muestra SELECCIONADA}

Para poder interpretar correctamente los datos obtenidos en las pruebas es necesario tener en cuenta las características de los centros y de las aulas; para ello es conveniente tener siempre presente el apartado que hace referencia a la muestra escogida para la realización del estudio.

El Instituto Duques de Nájera tiene una antigüedad de diez años y se encuentra ubicado en una vía de salida de la ciudad, está sometido a niveles de ruido exterior relativamente altos por su cercanía a la vía del tren y dado que la fachada principal recae a una calle en la que existe un tráfico medio/alto. El aula de música da a esta calle principal y se encuentra en el primer piso del edificio. Es pequeña: 50,31 metros cuadrados de planta y 173,5 metros cúbicos. Las paredes tienen colocadas hasta media altura baldosas pequeñas y el resto es yeso al igual que el techo. El suelo es de baldosas grandes pequeñas y el resto es yeso al igual que el techo.

El Instituto Escultor Daniel tiene una antigüedad de dieciocho años y se encuentra ubicado en uno de los barrios periféricos de la ciudad, en la zona oeste, junto a un ambulatorio. El número de edificaciones está aumentando y el tráfico rodado también. Todo el edificio recae en calles con elevado tráfico. El aula de música da a una rotonda y se encuentra en la planta baja, hecho por el que las condiciones acústicas son extraordinariamente negativas. Tiene 55,09 metros cuadrados de planta y 161,4 metros cúbicos. El suelo del aula es de baldosas grandes y las paredes y el techo de lucido de yeso. El profesor de música del centro opina que el aula es manifiestamente mejorable. 
El Instituto Hermanos D`Elhuyar tiene una antigüedad de veintinueve años y está ubicado en el centro de la ciudad. Se encuentra en una zona tranquila. El aula de música que da a un patio interior, se encuentra en el segundo piso del edificio; tiene 58,4 metros cuadrados de planta, 189,8 metros cúbicos y está insonorizada. El suelo del aula es de baldosas grandes. Las paredes tienen baldosas pequeñas hasta la mitad y el resto lucido de yeso, al igual que el techo.

El Instituto Batalla de Clavijo tiene una antigüedad de treinta y dos años y se encuentra ubicado en uno de los barrios periféricos de la ciudad. Es una zona tranquila. El aula de música da a un patio interior, se encuentra en el primer piso; y tiene doble ventana. Sus dimensiones son de 75,86 metros cuadrados de planta y de 238,2 metros cúbicos. Es una aula espaciosa y con mucha iluminación. El suelo del aula es de baldosas grandes. Las paredes tienen baldosas pequeñas hasta un metro de altura y el resto lucido de yeso, al igual que el techo.

\section{ReHABILITACIÓN ACÚSTICA}

Tras el estudio llevado a cabo en las aulas de música de cuatro centros de Educación Secundaria Obligatoria era necesario decidir qué aula iba a ser la que recibiera la mejora acústica. Las dos aulas con peores condiciones acústicas eran la del I.E.S. Duques de Nájera y la del I.E.S. Batalla de Clavijo.

Como el aula de música del Instituto Duques de Nájera está mal ubicada y es de dimensiones reducidas se decide no realizar una rehabilitación acústica ya que lo que prima es que se busque en el centro un aula más espaciosa y mejor ubicada.

Descartada el aula del I.E.S. Duques de Nájera se hace un estudio detallado del aula de música del I.E.S. Batalla de Clavijo que tiene buenas dimensiones y está muy bien ubicada. El aula tiene estructura de hormigón y forjado cerámico. Con 76,86 metros cuadrados, su forma es rectangular, está enlucida y pintada y tiene grandes ventanales en uno de sus lados. El aula está dotada de mobiliario clásico para el aula de música, con un armario de fábrica y sin ningún tipo de material absorbente.

Una vez conocidos todos los datos del aula y analizados detenidamente los resultados de las medidas Ilevadas a cabo en la investigación, se comunica al centro la necesidad de llevar a cabo una mejora de acondicionamiento acústico en el aula de música. El centro está de acuerdo en que el aula de música necesita la realización de la mejora y se acuerda hacerlo en la semana después de las vacaciones de Semana Santa. La única observación por parte de la dirección del centro es que el presupuesto no sobrepase los 3.000 euros.

Se piden tres presupuestos a dos empresas de Logroño especializadas en el acondicionamiento acústico de recintos y en sistemas de techos desmontables. Ambos presupuestos coinciden en que la solución para mejorar el aula es la colocación de un falso techo, ya que es la forma más eficaz, fácil y rápida de conseguir buenos resultados en una rehabilitación acústica (Recuero, 1999).

La empresa GETEIN (General de techos e instalaciones, S.L.) propone un presupuesto de colocación de falso techo desmontable de fibra fisurado modulo $60 * 60$ con perfilería vista lacada en blanco y una capa de fibra de roca de $40 \mathrm{~mm}$ de espesor $\left(76 \mathrm{~m}^{2}\right.$ a $18,9 \mathrm{E} / \mathrm{m}^{2}=1.436,4$ euros$)$. El presupuesto no es elevado pero el techo 
acústico que propone es el Ilamado fissured que muestra los siguientes coeficientes de absorción: a 125 hercios, 0,27; a 250 hercios, 0,32; a 500 hercios, 0,33; a 1.000 hercios, 0,43; a 2.000 hercios, 0,53 y a 4.000 hercios, 0,61. Según estos datos los coeficientes de absorción no son muy elevados sobre todo en las bajas frecuencias, es decir, a 125 y 250 hercios, que es donde el aula más lo necesita. Esta es la principal causa por la cual no es aceptado este presupuesto.

La empresa Ardanza y Colina, S.L. propone dos presupuestos: el primero más caro y completo; el segundo más barato e incompleto. El segundo presupuesto es igual al primero pero sin incluir la instalación del aglomerado de espuma en las vigas.

Como el primer presupuesto es el más completo y la dirección del centro no puso objeciones es el que se ha realizado. En la factura final, además de las dos cantidades anteriormente mencionadas, se incluye la retirada y colocación de lámparas fluorescentes por un importe de 118,7 euros y el I.V.A. del 16\%.

El importe total de la mejora acústica fue de 3.381,91 euros (562.703 pesetas).

El procedimiento que se lleva a cabo es la proyección en las dos vigas colgadas y en las dos medias de la cola de impacto así como en el aglomerado de espumas por una de sus caras, forrándose las mismas en su totalidad ( 3 caras en un caso y 2 en otro). Posteriormente se proyecta la cola sobre el aglomerado y sobre la placa Gyptone, igualmente forrando las vigas forradas en su totalidad. A continuación se procede a colocar el falso techo desmontable en los tres huecos que forman las vigas descolgadas, rebajando su altura desde el forjado a techo terminado en 17 centímetros.

La descripción de los materiales empleados es la siguiente:

- Placa de cartón-yeso ranurada tipo Gyptone con velo de fibra de vidrio adherida en su cara interior y de 13 milímetros de espesor. La curva de absorción del Gyptone con cámara de aire de 0,1 y fibra mineral de 0,08 muestra los siguientes coeficientes de absorción: a 125 hercios 0,45; a 250 hercios 0,92; a 500 hercios 0,85 ; a 1.000 hercios 0,7 y a 2.000 y 4.000 hercios 0,6 .

- Placa fibras minerales de medidas $1.200 * 600 * 19$ con ranuras en su longitud de 15 centímetros a ejes, clasificación al fuego M0. Este falso techo es conocido en el mercado con el nombre de Favia, Favia Face Cut y Illusion designs. Este material tiene un coeficiente de reducción de ruido del 0,7. Los datos de las pruebas en decibelios de este material para el aislamiento acústico es de 29 decibelios. El coeficiente de absorción global para todas las frecuencias es de 0,75 , a 125 hercios 0,45 ; a 250 y 500 hercios 0,6 ; a 1.000 y 2.000 hercios 0,8 y a 4.000 hercios 0,65 . La elección de este techo es porque tiene un alto coeficiente de absorción en las bajas frecuencias que es donde más hincapié hay que hacer; en las frecuencias medias aumenta el coeficiente y baja a 4.000 hercios, dato interesante para no perder en expresividad, aspecto importante para la actividad musical.

- Aglomerado de espumas de poliuretano de 40 milímetros de espesor y 80 kilogramos por metro cúbico de densidad, clasificación al fuego M1. La curva de absorción muestra los siguientes coeficientes: a 125 hercios 0,36; a 250 hercios 0,77 ; a 500 hercios 1 ; a 1.000 hercios 1,11; a 2.000 hercios 1,16 y a 4.000 hercios 1,13. 
- Perfilería acero galvanizado lacado blanco de 24 milímetros de ancho tanto perfiles primarios como secundarios.

Para la realización de los trabajos se han empleado los siguientes medios:

- Cola de impacto sin disolventes ni acetonas para el aglomerado de espumas y la placa Gyptone.

- Varilla M6, taco latón, paraguas y cuelgue universal PS-20 para perfilería T24 que sujeta la placa de fibras.

Una vez terminados los trabajos se procede de nuevo a realizar las mediciones en el aula de música. Mediciones psicoacústicas como acústicas.

La primera impresión, una vez terminada la rehabilitación acústica del aula de música, es que se ha producido una gran mejoría en el rendimiento acústico del aula, tanto en el coeficiente de reducción de ruido (NRC) como en el coeficiente de absorción $(\alpha)$ (Díaz, 1997).

Ahora queda comprobar a través de las mediciones si los resultados obtenidos en las distintas variables y parámetros han mejorado después de la mejora acústica del aula.

\section{ReSUltados}

Para que los resultados obtenidos se entiendan más claramente se van a exponer de forma desmembrada. En primer lugar se citan los resultados obtenidos a través de las pruebas de inteligibilidad de la palabra y después los obtenidos mediante las pruebas físico-acústicas (Jordan, 1977).

La prueba de inteligibilidad de la palabra se lleva a cabo con dos grupos de la Educación Secundaria Obligatoria y en las mismas condiciones que antes de realizar la mejora. En las siguientes tablas se pueden apreciar cambios importantes. El aumento de aciertos de palabras es muy considerable en todos los bloques pero especialmente en los primeros, es decir, los de menor intensidad, como se puede observar en el siguiente gráfico.

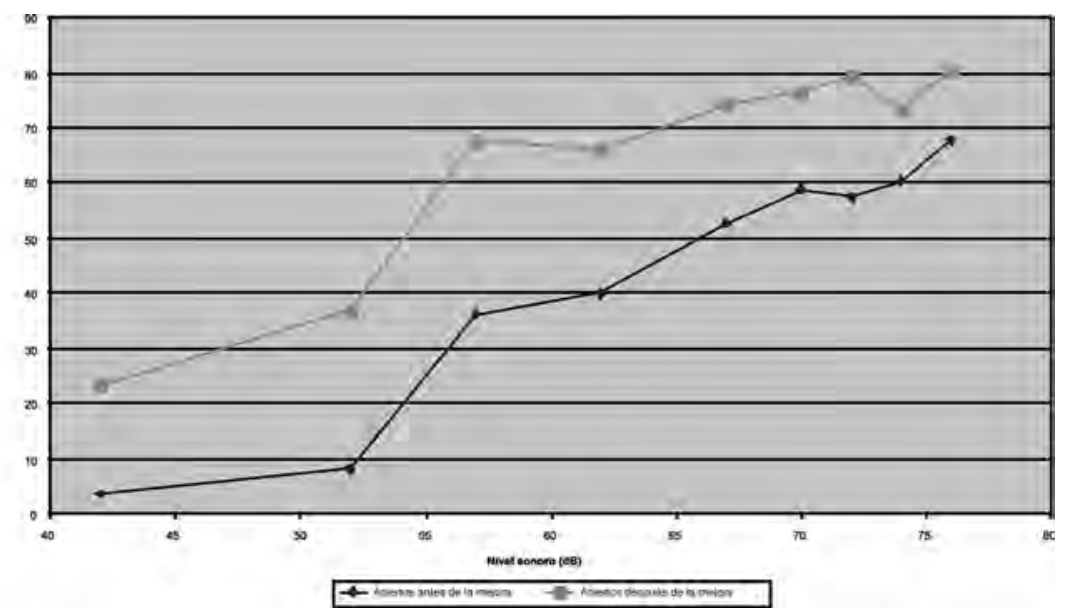


Los resultados de las medidas físicas de tiempos de reverberación para cada frecuencia, después de la rehabilitación acústica del aula, presentan una disminución muy considerable especialmente en las bajas frecuencias.

En todas las frecuencias los tiempos de reverberación no superan los 0,6 segundos con niños. Esta disminución va a contribuir a alcanzar el confort acústico del aula.

\section{Conclusiones}

El aula de música del I.E.S. Batalla de Clavijo, antes de la rehabilitación acústica, tenía malas condiciones acústicas según la información extraída de los valores de la mayoría de los parámetros estudiados, excepto en el ruido exterior ya que es el aula que menor ruido exterior tiene que soportar. El aula cuenta con las mayores dimensiones respecto a las otras aulas de música.

El test de inteligibilidad de la palabra no proporciona malos resultados en los primeros bloques de palabras porque como el ruido exterior es mínimo esto hace buena la audición cuando la intensidad sonora es baja. A medida que aumenta la intensidad los alumnos empiezan a tener mayores problemas respecto a los de las otras aulas porque entran en juego otros parámetros no tan favorables. Como consecuencia de esto el umbral de inteligibilidad es alcanzado a 66,5 decibelios. Esto indica que los alumnos consiguen escuchar el 50\% total de audición a un elevado número de decibelios.

En la citada aula, parámetros como tiempo de reverberación, RASTI, claridad acústica, tiempo de decaimiento primario y tiempo de elevación marcaban índices muy poco favorables para conseguir una buena inteligibilidad tanto para la música como para la palabra. En todos los parámetros acústicos mencionados alcanza los peores resultados y con diferencia en relación a las otras aulas de música del estudio. Con estos datos y teniendo en cuenta que es un aula muy bien ubicada y con buenas dimensiones es la propuesta para realizar un acondicionamiento acústico que consiga mejorar los parámetros citados anteriormente.

Una vez realizada la mejora del aula de música del I.E.S. Batalla de Clavijo se observan cambios acústicos importantes. El test de inteligibilidad proporciona datos muy diferentes a los conseguidos antes; es decir, en todos los bloques de palabras se superan ampliamente los aciertos de palabras y es más también supera en todos los bloques a los aciertos de las otras aulas de música. El umbral de inteligibilidad pasa de estar en los 66,5 decibelios a los 54 decibelios, es decir, por debajo del alcanzado por el I.E.S. Hermanos D’Elhuyar que obtuvo el mejor resultado con 56 decibelios.

Después de la rehabilitación acústica del aula todos los parámetros han mejorado excepto el ruido de fondo que aumenta débilmente debido a la existencia esporádica de un ruido procedente de las tuberías. El ruido de fondo ponderación " $\mathrm{A}$ " pasa de estar en 27 decibelios a 34,6 decibelios; y el ruido total (L) pasa de los 42,4 decibelios a los 53 decibelios. Como anteriormente se ha expuesto, la OMS propone 35 decibelios " $\mathrm{A}$ " de ruido en las aulas, por tanto, este límite no se ha rebasado.

Los tiempos de reverberación han descendido considerablemente después de la mejora tanto con como sin niños y en todas las frecuencias. Por ejemplo, si el tiem- 
po de reverberación calculado con medidas físicas a 500 hercios y con niños era de 0,92 ahora es de 0,49 ; y a 2.000 hercios antes era de 0,78 y ahora de 0,45. Estos tiempos siguen siendo más altos que los establecidos en la normativa finlandesa.

EI RASTI con niños ha aumentado; si antes de la mejora era de 0,63 ahora es de 0,78 . Este valor se encuentra en el margen propuesto por el Ministerio de Medio Ambiente Finlandés que es de 0,72 - 0,81.

La claridad acústica pasa de ser 0,3312 a 0,45179. La claridad expresa sensación de sonido directo y este aspecto es muy importante para la inteligibilidad de la palabra y la música. Hay que conseguir una claridad producida por sonido directo o por sus primeras reflexiones ya que si no se aumenta el tiempo de reverberación y con ello disminuye la inteligibilidad.

El tiempo de decaimiento primario pasa de 286,1 milisegundos a 192,3 milisegundos. La caída del sonido ha sido más rápida después de la rehabilitación acústica y por consiguiente la inteligibilidad en el aula será buena.

El tiempo de elevación de los sonidos pasa de 80 milisegundos a 52,68 milisegundos, después de la mejora. Este descenso de milisegundos va en la buena dirección de obtener una mejor inteligibilidad dentro del aula de música.

Después de la rehabilitación acústica del aula de música del Instituto de Educación Secundaria Batalla de Clavijo, los valores de los diferentes parámetros han experimentado un cambio considerable en orden a alcanzar el confort acústico deseado para llevar a cabo las actividades propias de la asignatura de música dentro del aula.

\section{REFERENCIAS BIBLIOGRÁFICAS}

ALONSO, J. (2000). Acústica musical: un puente o una simbiosis entre la física y la música. Eufonía. Didáctica de la Música, 18, 43-54.

DELGADO, C. (1995). Inteligibilidad de la palabra en la medida de la audición. Actas del Simposio sobre Acústica Fisiológica y Audiometría (pp. 27-36). Santiago de Compostela.

DÍAZ, C. (1997). Sobre la utilización de los coeficientes de absorción de los materiales en la expresión de Eyring del tiempo de reverberación de una sala. Revista de Acústica, 28 (3-4), 66-67.

JORDAN, V. L. (1977). Acoustical criteria and acoustical qualities of concert hall. Music, Room and Acoustics, 17, 114-123.

KINSLER, L. y FREY, A. (1988). Fundamentos de acústica. México: Limusa.

PIERCE, J. R. (1985). Los sonidos de la música. Barcelona: Labor.

RECUERO, M. (1999). Acústica arquitectónica aplicada. Madrid: Paraninfo. 